\title{
REMARKS ABOUT THE DIGITS OF INTEGERS
}

\author{
RALPH ALEXANDER ${ }^{1}$ \\ (Received 6 January 1969; revised 23 September 1969) \\ Communicated by B. Mond
}

\section{Introduction}

Let $n_{1}<n_{2}<\cdots$ be the sequence of positive integers whose base $b$ representations involve the digit $t \leqq b-1$ at most $d-1$ times. B. D. Craven [2] shows that $\sum_{i} 1 / n_{i}$ converges by giving an upper bound for this sum as a function of $b$ and $d$.

The method used by Professor Craven gives an upper bound on the sum $\sum_{i} 1 / 0_{i}$, where $0_{1}<0_{2}<\cdots$ is the much larger positive integer sequence whose members have the property that the digit $t$ occurs at most $d-1$ times in succession in their base $b$ representations. The first theorem is a corollary to the main theorem in [2].

THEOREM 1. Let $0_{1}<0_{2}<\cdots$ be the sequence of positive integers whose base $b$ representations involve the digit $t \leqq b-1$ at most $d-1$ times in succession. Then $\sum_{i} 1 / 0_{i}$ is less than $b^{d}(1+d \log b)$.

Using a variation of one of the methods in our article [1], we will give estimates of both types of sum mentioned above.

\section{A method of estimating certain harmonic sums}

Let $n=\sum_{r=0}^{\infty} a(n, r) b^{r}$ be the representation of $n$ to the base $b$. We define $L(n)$ to be $k+1$, where $k$ is the largest integer $i$ for which $a(n, i) \neq 0$. Clearly $L(n)$ is the number of significant digits in the base $b$ representation of $n$. For any positive integer $n$,

$$
n<b^{L(n)} \leqq b n .
$$

If $\left\{a_{i}+j c_{i}: j=0,1, \cdots\right\}, i=1,2, \cdots$, is a pairwise disjoint sequence of arithmetic progressions in the positive integers, then

$$
\sum_{i} 1 / c_{i} \leqq 1
$$

1 This research was supported by NSF grant gp-7537. 
This follows at once from the observation that the natural density of the $i$-th progression is $1 / c_{i}$.

THEOREM 2. Let $n_{1}<n_{2}<\cdots$ be the sequence of positive integers whose base $b$ representations involve the digit $t \leqq b-1$ at most $d-1$ times. Then $\sum_{i} 1 / n_{i}$ is at most $d b^{2}$.

ProOf. Let $m_{1}<m_{2}<\cdots$ be the sequence of positive integers whose base $b$ representations involve the digit $t$ exactly $k$ times. We claim that the sequence of arithmetic progressions

$$
\left\{\left[m_{i}+t b^{L\left(m_{i}\right)}\right]+j b^{L\left(m_{i}\right)+1}: j=0,1, \cdots\right\}, \quad i=1,2, \cdots
$$

is pairwise disjoint. Indeed, given any integer in the union of these progressions, the associated $m_{i}$ is immediately identified by noting where the $(k+1)$-st $t$ occurs in the base $b$ representation of that integer. For example (using arabic notation with $b=10, t=0$, and $k=2$ ), suppose the integer is $103,020,109$; then our method of construction would give $m_{i}$ as 20,109 . Hence, $\sum_{i} b^{-L\left(m_{i}\right)-1} \leqq 1$ by remark (2) and by remark (1) it follows that $\sum_{i} 1 / m_{i} \leqq b^{2}$. Letting $k=0,1, \cdots$, $d-1$ and summing, we obtain $\sum_{i} 1 / n_{i} \leqq d b^{2}$.

THEOREM 3. Let $0_{1}<0_{2}<\cdots$ be the sequence of positive integers whose base $b$ representations do not have the digit $t \leqq b-1$ appearing $d$ times in succession. Then

$$
\sum_{i} 1 / 0_{i} \leqq b^{d+2} .
$$

Proof. We consider the sequence of progressions

$$
\left\{\left[0_{i}+\sum_{k=1}^{d} t b^{L\left(0_{i}\right)+k}\right]+j b^{L\left(0_{i}\right)+d+1}: j=0,1, \cdots\right\}, \quad i=1,2, \cdots .
$$

As before, these progressions are pairwise disjoint, since an integer in the union of the progressions may be associated with a unique $0_{i}$ simply by examining the blocks of $t$ 's in the integer's base $b$ representation. For example (using arabic notation with $b=10, t=9$, and $d=3$ ), suppose the integer is $699,909,927$; then our method of construction identifies $0_{i}$ as 9,927 .

Thus

be remark (2), and

$$
\sum_{i} b^{-L\left(0_{i}\right)-d-1} \leqq 1
$$

$$
\sum_{i} 1 / 0_{i} \leqq b^{d+2}
$$

by remark (1).

\section{Closing remarks}

By a more careful estimate of the sums occurring in the proof of Theorem 2, we could replace $d b^{2}$ by $K d b \log b$, where $K$ is a constant slightly larger than 1 . 
Also, we note that Theorem 3 improves Theorem 1 only if $d \log b>b^{2}$. Assuming $t=0$ and using a more elaborate estimate, we could replace $b^{d+2}$ by $K b^{d} \log b$ in Theorem 3.

Finally, we wish to thank the referee for a number of comments which helped improve the accuracy and clarity of our article.

\section{References}

[1] R. Alexander, 'Density and digits of sequences of integers', Michigan Math, $J .16$ (1969), 85-92.

[2] B. D. Craven, 'On digital distribution in some integer sequences', J. Australian Math. Soc. 5 (1965), 325-330.

University of Illinois 61801 